\title{
KLT DEL PEZZO SURFACES WHICH ARE NOT GLOBALLY F-SPLIT
}

\author{
PAOLO CASCINI, HIROMU TANAKA, JAKUB WITASZEK
}

\begin{abstract}
We construct a klt del Pezzo surface which is not globally $F$-split, over any algebraically closed field of positive characteristic.
\end{abstract}

\section{Contents}

1. Introduction

2. Preliminary Results 3

2.1. Notation 3

2.2. Preliminaries 4

3. F-purity on the F-pure threshold 6

4. Non-globally-F-split klt del Pezzo surfaces 10

5. Non-F-pure canonical threefolds 14

References 17

\section{INTRODUCTION}

It has been clear for a long time that the right category of singularities to work with, in trying to run the minimal model programme in characteristic zero, is the category of Kawamata log terminal pairs klt, for short.

The main difficulty in trying to extend the classic results in minimal model programme, such as the base point free theorem and the cone theorem, to any projective variety defined over an algebraically closed field of positive characteristic is the fact that the Kawamata-Viehweg vanishing theorem does not hold in this generality. On the other hand, over the last few years, many methods have been developed to use the Frobenius morphism to lift sections, as a replacement of the vanishing

2010 Mathematics Subject Classification. 14E30, 13A35.

Key words and phrases. Log del Pezzo surface, F-singularity, positive characteristic.

All three authors were funded by EPSRC. 
theorems. As a consequence, $F$-singularities are more suitable singularities to work with in terms of generalising some of the results in birational geometry to varieties defined over a field of positive characteristic. These singularities were introduced in [HH90] to develop new techniques in commutative algebra.

The recent progress in the field has been sparked by the introduction of strongly $F$-regular singularities and globally $F$-regular varieties ([Smi00] and [SS10]). Those notions are pereceived as correct counterparts of klt singularities and klt Fano varieties, respectively. For example, Watanabe showed that over an algebraically closed field of characteristic $p>5$, any one dimensional log Fano pair $(X, \Delta)$ with standard coefficients is globally $F$-regular [Wat91, Theorem 4.2]. This result played an important role in the work of Hacon and $\mathrm{Xu}$, who showed the existence of minimal models for any projective $\mathbb{Q}$-factorial terminal threefold with pseudo-effective canonical divisor, defined over an algebraically closed field of characteristic $p>5$ [HX15].

Later on, the first author, together with Gongyo and Schwede, showed that a two-dimensional klt singularity $(X, \Delta)$ is strongly $F$-regular for big enough characteristic depending only on the coefficients of $\Delta$ CGS14.

The goal of this paper is to show that the natural generalization of the results in [Har98a, Wat91, CGS14] do not hold true even under the assumption that the boundary divisor is trivial (cf. [PST14, Question 5.23]). Our main result is:

Theorem 1.1. Over any algebraically closed field $k$ of characteristic $p>0$, there exists a projective Kawamata log terminal surface $X$ such that $-K_{X}$ is ample and $X$ is not globally $F$-split.

Note that, if $X$ is a smooth del Pezzo surface over an algebraically closed field of characteristic $p>5$, then $X$ is globally $F$-regular [Har98a, Example 5.5]. In [CTW15, we show that any Kawamata log terminal del Pezzo surface over an algebraically closed field of large characteristic which is not globally $F$-regular, admits a log resolution which is liftable to characteristic zero.

By considering the cone over a klt del Pezzo surface which is not globally $F$-split, as in Theorem 1.1, we obtain a three-dimensional klt singularity in arbitrary characteristic which is not $F$-pure. On the other hand, we give a direct and simpler construction of such singularities. In particular, the varieties that we construct, admit only canonical hypersurface singularities. 
Theorem 1.2. Over any algebraically closed field $k$ of characteristic $p>0$, there exists a three-dimensional canonical variety $X$, which is not F-pure.

Theorem 1.1 and Theorem 1.2 are proved in Section 4 and Section 5 , respectively.

Acknowledgement: We would like to thank Y. Gongyo, Y. Kawamata, Z. Patakfalvi, A. Sannai, K. Schwede and S. Takagi for many useful discussions and comments.

\section{Preliminary Results}

2.1. Notation. We work over an algebraically closed field $k$ of characteristic $p>0$, unless otherwise mentioned. We say that a scheme $X$ is of characteristic $p>0$ if the natural morphism $X \rightarrow \operatorname{Spec} \mathbb{Z}$ factors through Spec $\mathbb{Z} / p \mathbb{Z}$. For any prime $p$, we denote by $\mathbb{Z}_{(p)}$ the set of rational numbers whose denominator is not divisible by $p$.

We say that $X$ is a variety over an algebraically closed field $k$, if $X$ is an integral scheme which is separated and of finite type over $k$. A curve is a variety of dimension one, and a surface is a variety of dimension two.

We refer to [KM98] for the classical definitions of singularities (for example klt, plt, dlt and log canonical) appearing in the minimal model programme. For a $\mathbb{Q}$-Cartier $\mathbb{Q}$-divisor $D$ on a normal variety, the Cartier index of $D$ is the smallest positive integer $m$ such that $m D$ is Cartier.

Given a scheme $X$ of characteristic $p>0$, we denote by $F: X \rightarrow X$ the absolute Frobenius morphism, and we say that $X$ is $F$-finite if $F_{*} \mathcal{O}_{X}$ is a finitely generated $\mathcal{O}_{X}$-module. We now recall the definitions of some classes of $F$-singularities (e.g. see [SS10, Definition 3.1]):

Definition 2.1. Let $X$ be an $F$-finite normal scheme of characteristic $p>0$ (for example a normal variety over an algebraically closed field) and let $\Delta$ be an effective $\mathbb{R}$-divisor on $X$.

(1) The pair $(X, \Delta)$ is globally $F$-split if for every $e \in \mathbb{Z}_{>0}$, the natural map

$$
\mathcal{O}_{X} \stackrel{F^{e}}{\longrightarrow} F_{*}^{e} \mathcal{O}_{X} \hookrightarrow F_{*}^{e} \mathcal{O}_{X}\left(\left\llcorner\left(p^{e}-1\right) \Delta\right\lrcorner\right)
$$

splits as an $\mathcal{O}_{X}$-module homomorphism.

(2) The pair $(X, \Delta)$ is globally sharply $F$-split if there exists $e \in \mathbb{Z}_{>0}$, such that the natural map

$$
\mathcal{O}_{X} \stackrel{F^{e}}{\longrightarrow} F_{*}^{e} \mathcal{O}_{X} \hookrightarrow \underset{3}{F_{*}^{e}} \mathcal{O}_{X}\left(\left\ulcorner\left(p^{e}-1\right) \Delta\right\urcorner\right)
$$


splits as an $\mathcal{O}_{X}$-module homomorphism.

(3) The pair $(X, \Delta)$ is globally $F$-regular if for every effective divisor $E$ on $X$, there exists $e \in \mathbb{Z}_{>0}$ such that the natural map

$$
\mathcal{O}_{X} \stackrel{F^{e}}{\rightarrow} F_{*}^{e} \mathcal{O}_{X} \hookrightarrow F_{*}^{e} \mathcal{O}_{X}\left(\left\llcorner\left(p^{e}-1\right) \Delta\right\lrcorner+E\right)
$$

splits as an $\mathcal{O}_{X}$-module homomorphism.

(4) The pair $(X, \Delta)$ is $F$-pure (resp. sharply $F$-pure, strongly $F$ regular) if (Spec $\mathcal{O}_{X, x},\left.\Delta\right|_{\text {Spec } \mathcal{O}_{X, x}}$ ) is globally $F$-split (resp. globally sharply $\mathrm{F}$-split, globally $F$-regular) for every point $x \in X$.

If the coefficients of $\Delta$ belong to $\mathbb{Z}_{(p)}$, then being sharply $F$-split coincides with being $F$-split.

2.2. Preliminaries. We begin by recalling the following basic result:

Lemma 2.2. Let $f: X \rightarrow Y$ be a proper birational morphism of normal varieties. Let $\Delta$ be an effective $\mathbb{Q}$-divisor on $X$. If $(X, \Delta)$ is globally $F$-split, then so is $\left(Y, f_{*} \Delta\right)$.

Proof. Let $e \in \mathbb{Z}_{>0}$. Since $(X, \Delta)$ is globally $F$-split, the Frobenius homomorphism induced by $(X, \Delta)$ splits:

$$
\text { id : } \mathcal{O}_{X} \stackrel{F^{e}}{\longrightarrow} F_{*}^{e} \mathcal{O}_{X}\left(\left\llcorner\left(p^{e}-1\right) \Delta\right\lrcorner\right) \stackrel{\exists \varphi}{\longrightarrow} \mathcal{O}_{X}
$$

Consider an open subscheme $j: U \hookrightarrow Y$ of codimension at least two, such that the restriction of $f$ to $U$ induces an isomorphism $f^{-1}(U) \rightarrow U$. By restricting to $U$, we have that the Frobenius homomorphism splits on $U$ :

$$
\mathrm{id}: \mathcal{O}_{U} \stackrel{F^{e}}{\longrightarrow} F_{*}^{e} \mathcal{O}_{U}\left(\left\llcorner\left(p^{e}-1\right) f_{*} \Delta\right\lrcorner\right) \stackrel{\left.\varphi\right|_{U}}{\longrightarrow} \mathcal{O}_{U} .
$$

Since $U$ is of codimension at least two, the Lemma follows by taking the pushforward by $j_{*}$.

By a result of Hacon and $\mathrm{Xu}$, also the opposite implication holds true:

Lemma 2.3 ([HX15, Prop. 2.11]). Let $(Y, \Delta)$ be a globally F-regular pair and $f: X \rightarrow Y$ a proper birational morphism of normal varieties such that $f^{*}\left(K_{Y}+\Delta\right)=K_{X}+\Delta^{\prime}$, for some $\mathbb{Q}$-divisor $\Delta^{\prime} \geq 0$.

Then, $\left(X, \Delta^{\prime}\right)$ is globally $F$-regular.

The following result is a consequence of Wat91 and an elementary calculation. 
Lemma 2.4. Let $k$ be an algebraically closed field of characteristic $p>0$. Then, there exist four distinct closed points $Q_{1}, \ldots, Q_{4} \in \mathbb{P}_{k}^{1}$ such that the pair

$$
\left(\mathbb{P}_{k}^{1}, \frac{1}{2} \sum_{i=1}^{4} Q_{i}\right)
$$

is not globally F-split.

Proof. If $p=2$, then [Wat91, Thm. 4.2(h)] implies that the pair is not globally $F$-split, for any choice of the points $Q_{1}, \ldots, Q_{4}$. Thus, we may write $p=2 n+1$ for some $n \in \mathbb{Z}_{>0}$. Consider the four distinct points of $\mathbb{P}_{k}^{1}$ :

$$
Q_{1}=[1,0], Q_{2}=[0,1], Q_{3}=[-1,1], \text { and } Q_{4}=[-\mu, 1]
$$

where $\mu \in k \backslash\{0,1\}$. By [Wat91, Thm. 4.2(h)], the pair

$$
\left(\mathbb{P}_{k}^{1}, \frac{1}{2} \sum_{i=1}^{4} Q_{i}\right)
$$

is globally $F$-split if and only if the coefficient of $x^{n}$ in the expansion of $(x+1)^{n}(x+\mu)^{n}$ is not zero.

The coefficient of $x^{n}$ can be expressed in terms of the following monic polynomial in the variable $\mu$ :

$$
\sum_{i=0}^{n}\left(\begin{array}{l}
n \\
i
\end{array}\right)\left(\begin{array}{c}
n \\
n-i
\end{array}\right) \mu^{i}=\mu^{n}+n^{2} \mu^{n-1}+\ldots+1 \in k[\mu] .
$$

Since $k$ is algebraically closed, it is enough to show that such a polynomial admits a solution which is different from zero and one. By contradiction, assume that

$$
\mu^{n}+n^{2} \mu^{n-1}+\ldots+1=\mu^{a}(\mu-1)^{b},
$$

for some non-negative integers $a$ and $b$ satisfying $a+b=n$. Clearly $a=0$, hence we obtain

$$
\mu^{n}+n^{2} \mu^{n-1}+\ldots+1=(\mu-1)^{n} \text { in } k[\mu] .
$$

It follows that $n^{2} \equiv-n \bmod p$ and, in particular, $p$ divides either $n$ or $n+1$. Since $n+1<2 n+1=p$, we get a contradiction. Thus, the claim follows.

Let $X$ be a normal surface over an algebraically closed field $k$. Let $C$ be a smooth prime divisor on $X$ such that $(X, C)$ is $\log$ canonical. Then there exists an effective $\mathbb{Q}$-divisor $\operatorname{Diff}_{C}$ on $C$, called the different, such that

$$
\left.\left(K_{X}+C\right)\right|_{C}=K_{5}+\operatorname{Diff}_{C}
$$


[K+92, Prop.-Def. 16.5].

Lemma 2.5. Let $(Z, C)$ be a two-dimensional projective plt pair over an algebraically closed field of characteristic $p>0$, such that $C$ is a smooth prime divisor. If $\left(C, \mathrm{Diff}_{C}\right)$ is not globally $F$-split and the Cartier index of $K_{Z}+C$ is not divisible by $p$, then $(Z, C)$ is not globally F-split.

Proof. Let $e \in \mathbb{Z}_{>0}$ be such that $\left(p^{e}-1\right)\left(K_{Z}+C\right)$ is Cartier. It is enough to show that the trace map

$$
\operatorname{Tr}_{Z}^{e}: H^{0}\left(Z, F_{*}^{e} \mathcal{O}_{Z}\left(-\left(p^{e}-1\right)\left(K_{Z}+C\right)\right)\right) \rightarrow H^{0}\left(Z, \mathcal{O}_{Z}\right)
$$

is zero.

Let $\mathcal{L}:=\mathcal{O}_{Z}\left(-\left(p^{e}-1\right)\left(K_{Z}+C\right)\right)$. Then

$$
\left.\mathcal{L}\right|_{C}=\mathcal{O}_{C}\left(-\left(p^{e}-1\right)\left(K_{C}+\operatorname{Diff}_{C}\right)\right)
$$

Consider the following commutative diagram:

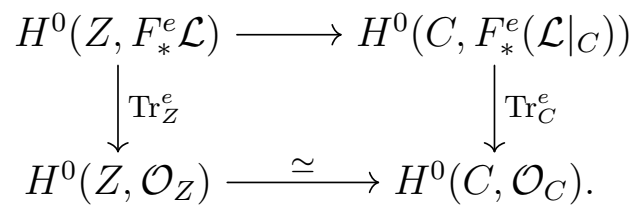

Since $\left(C, \operatorname{Diff}_{C}\right)$ is not globally $F$-split, the right vertical arrow $\operatorname{Tr}_{C}^{e}$ is zero. Hence, $\operatorname{Tr}_{Z}^{e}$ is zero, and the claim follows.

\section{F-PURity on the F-PURE THREshold}

The goal of this section is to prove Theorem 3.2, which implies that being globally $F$-split is preserved after taking a limit.

The result was already known in some special cases (e.g. see [Har06, Proposition 2.6], [Her12, Theorem 4.1] and [Sch08, Remark 5.5]). On the other hand, if we consider a pair $(X, \mathfrak{a})$ consisting of a normal variety $X$ and an ideal $\mathfrak{a}$, instead of a divisor, then the result does not hold [TW04, Proposition 2.2(6) and Remark 2.3(2)]. Moreover, note that being globally sharply $F$-split is not preserved after taking a limit [SS10, Remark 6.2].

We begin by recalling the definitions of the $F$-pure and the $F$-split thresholds. The first notion was introduced by Takagi and Watanabe in [TW04]. We refer to [BMS08, BSTZ10, HNBWZ15] for more results in this direction.

Definition 3.1. Let $X$ be an $F$-finite normal scheme of characteristic $p>0$ and let $\Delta$ and $D$ be effective $\mathbb{R}$-divisors. 
(1) We define the $F$-split threshold of $(X, \Delta)$ with respect to $D$ as $\operatorname{fst}(X, \Delta ; D):=\sup \left\{t \in \mathbb{R}_{\geq 0} \mid(X, \Delta+t D)\right.$ is globally $F$-split $\}$.

(2) We define the $F$-pure threshold of $(X, \Delta)$ with respect to $D$ as $\operatorname{fpt}(X, \Delta ; D):=\sup \left\{t \in \mathbb{R}_{\geq 0} \mid(X, \Delta+t D)\right.$ is $F$-pure $\}$.

The following Theorem is used in Section 4 to prove Theorem 1.1:

Theorem 3.2. Let $X$ be an F-finite noetherian normal scheme of characteristic $p>0$ and let $\Delta$ be an $\mathbb{R}$-divisor such that $(X, \Delta)$ is globally $F$-split. Let $D$ be an effective $\mathbb{R}$-divisor on $X$ and let $c=\operatorname{fst}(X, \Delta ; D)$.

Then $(X, \Delta+c D)$ is globally F-split.

Proof. If $c=0$, then there is nothing to show. Thus, we may assume $c>0$. We divide the proof into three steps:

Step 1. We first show the Theorem, assuming that the following property holds:

(1) $D$ and $\Delta$ do not have any common component, $D$ has coefficients in $\mathbb{R} \backslash \mathbb{Z}_{(p)}$, and fst $(X, \Delta ; D)=1$.

Let $e \in \mathbb{Z}_{>0}$. We need to show that

$$
\mathcal{O}_{X} \longrightarrow F_{*}^{e} \mathcal{O}_{X}\left(\left\llcorner\left(p^{e}-1\right)(\Delta+D)\right\lrcorner\right)
$$

splits. Since $D$ and $\Delta$ do not have any common component, we have

$$
\left\llcorner\left(p^{e}-1\right)(\Delta+D)\right\lrcorner=\left\llcorner\left(p^{e}-1\right) \Delta\right\lrcorner+\left\llcorner\left(p^{e}-1\right) D\right\lrcorner .
$$

Since the coefficients of $D$ are contained in $\mathbb{R} \backslash \mathbb{Z}_{(p)}$, there exists $t \in(0,1)$ such that

$$
\left\llcorner\left(p^{e}-1\right) D\right\lrcorner=\left\llcorner\left(p^{e}-1\right) t D\right\lrcorner .
$$

Since fst $(X, \Delta ; D)=1$, the claim follows.

Step 2. We now show the Theorem assuming that the following property holds:

(2) $D$ is a prime divisor, $D \not \subset \operatorname{Supp} \Delta$, and $\Delta$ has coefficients in $\mathbb{Z}_{(p)}$. Moreover, fst $(X, \Delta ; D) \in \mathbb{Z}_{(p)}$.

Since the coefficients of $\Delta+c D$ are contained in $\mathbb{Z}_{(p)}$, it is enough to show that there exists $e \in \mathbb{Z}_{>0}$ such that

$$
\mathcal{O}_{X} \longrightarrow F_{*}^{e} \mathcal{O}_{X}\left(\left\llcorner\left(p^{e}-1\right)(\Delta+c D)\right\lrcorner\right)
$$

splits. Consider the subset $N_{1} \subset \mathbb{Z}_{>0}$ such that $e \in N_{1}$ if and only if $\left(p^{e}-1\right) \Delta$ has coefficients in $\mathbb{Z}$ and $\left(p^{e}-1\right) c$ is an integer. For any $e \in N_{1}$, we define

$$
\nu(e):=\max \left\{s \in \mathbb{Z}_{\geq 0} \mid \mathcal{O}_{X} \rightarrow \underset{7}{F_{*}^{e}} \mathcal{O}_{X}\left(\left(p^{e}-1\right) \Delta+s D\right) \text { splits }\right\}
$$


By the definition of $\nu(e)$ and $c$, we see that

$$
\nu(e) \leq\left(p^{e}-1\right) c \leq \nu(e)+1 .
$$

Thus,

$$
\nu(e) \in\left\{\left(p^{e}-1\right) c-1,\left(p^{e}-1\right) c\right\} .
$$

If $\nu(e)=\left(p^{e}-1\right) c$ for some $e \in N_{1}$, then $(X, \Delta+c D)$ is globally $F$-split and we are done. We now assume by contradiction that

$$
\nu(e)=\left(p^{e}-1\right) c-1 .
$$

for every $e \in N_{1}$.

Pick $e \in N_{1}$. Then, $2 e \in N_{1}$. Consider

$$
\mathcal{O}_{X} \rightarrow F_{*}^{e} \mathcal{O}_{X}\left(\left(p^{e}-1\right) \Delta\right) \text {. }
$$

After tensoring by $\mathcal{O}_{X}\left(\left(p^{e}-1\right) \Delta+(\nu(e)+1) D\right)$, taking the double dual, and applying $F_{*}^{e}$, we obtain:

$F_{*}^{e} \mathcal{O}_{X}\left(\left(p^{e}-1\right) \Delta+(\nu(e)+1) D\right) \rightarrow F_{*}^{2 e} \mathcal{O}_{X}\left(\left(p^{2 e}-1\right) \Delta+p^{e}(\nu(e)+1) D\right)$.

Since

$$
\mathcal{O}_{X} \rightarrow F_{*}^{e} \mathcal{O}_{X}\left(\left(p^{e}-1\right) \Delta+(\nu(e)+1) D\right)
$$

does not split, neither does

$$
\mathcal{O}_{X} \rightarrow F_{*}^{2 e} \mathcal{O}_{X}\left(\left(p^{2 e}-1\right) \Delta+p^{e}(\nu(e)+1) D\right) .
$$

Thus,

$$
\nu(2 e)<p^{e}(\nu(e)+1) .
$$

Since $\nu(d)=\left(p^{d}-1\right) c-1$ for $d \in\{e, 2 e\}$, and since $\left(p^{e}-1\right) c \in \mathbb{Z}_{>0}$, we have

$\left(p^{2 e}-1\right) c-1<p^{e}\left(p^{e}-1\right) c \leq p^{e}\left(p^{e}-1\right) c+\left(p^{e}-1\right) c-1=\left(p^{2 e}-1\right) c-1$.

This is a contradiction. Thus, the claim follows.

Step 3. We now show the Theorem in the general case.

We may write

$$
\Delta+c D=\sum_{i=1}^{r} a_{i} A_{i}+\sum_{j=1}^{s} b_{j} B_{j}
$$

where $a_{i} \in \mathbb{Z}_{(p)}, b_{j} \in \mathbb{R} \backslash \mathbb{Z}_{(p)}$ and $A_{1}, \ldots, A_{r}, B_{1}, \ldots, B_{s}$ are irreducible components.

We first show that $\left(X, \sum_{i=1}^{r} a_{i} A_{i}\right)$ is globally $F$-split. If not, then by Step 2, we can find $a_{1}^{\prime} \in \mathbb{Z}_{(p)}$ such that $0 \leq a_{1}^{\prime}<a_{1}$ and $\left(X, a_{1}^{\prime} A_{1}+\right.$ $\left.\sum_{i=2}^{r} a_{i} A_{i}\right)$ is not globally $F$-split. Proceeding this way, for every $1 \leq$ 
$i \leq r$, we can find $a_{i}^{\prime} \in \mathbb{Z}_{(p)}$ such that $0 \leq a_{i}^{\prime}<a_{i}$ and $\left(X, \sum_{i=1}^{r} a_{i}^{\prime} A_{i}\right)$ is not globally $F$-split. In particular, there exists $\delta \in(0, c)$ such that

$$
\sum_{i=1}^{r} a_{i}^{\prime} A_{i} \leq \Delta+(c-\delta) D .
$$

This contradicts the fact that $(X, \Delta+(c-\delta) D)$ is globally $F$-split for any $\delta \in(0, c)$. Thus, $\left(X, \sum_{i=1}^{r} a_{i} A_{i}\right)$ is globally $F$-split.

Let

$$
c^{\prime}:=\operatorname{fst}\left(X, \sum_{i=1}^{r} a_{i} A_{i} ; \sum_{j=1}^{s} b_{j} B_{j}\right) .
$$

If $c^{\prime}>1$, then $(X, \Delta+c D)$ is globally $F$-split, by the definition of the $F$-split threshold. If $c^{\prime}=1$, then the same claim follows from (2) of Step 1.

We assume now by contradiction that $0 \leq c^{\prime}<1$. For each $j=$ $1, \ldots, s$, there exists $b_{j}^{\prime} \in \mathbb{Z}_{(p)}$ such that $c^{\prime} b_{j}<b_{j}^{\prime}<b_{j}$. In particular, the pair

$$
\left(X, \sum_{i=1}^{r} a_{i} A_{i}+\sum_{j=1}^{s} b_{j}^{\prime} B_{j}\right)
$$

is not globally $F$-split. By Step 2, it follows that

$$
\left(X, a_{1}^{\prime} A_{1}+\sum_{i=2}^{r} a_{i} A_{i}+\sum_{j=1}^{s} b_{j}^{\prime} B_{j}\right)
$$

is not globally $F$-split for some $a_{1}^{\prime} \in \mathbb{Z}_{(p)}$ with $0 \leq a_{1}^{\prime}<a_{1}$. Proceeding this way, we see that

$$
\left(X, \sum_{i=1}^{r} a_{i}^{\prime} A_{i}+\sum_{j=1}^{s} b_{j}^{\prime} B_{j}\right)
$$

is not globally $F$-split for some $a_{i}^{\prime} \in \mathbb{Z}_{(p)}$ with $0 \leq a_{i}^{\prime}<a_{i}$ for $i=$ $1, \ldots, r$. We may find $\delta>0$ such that:

$$
\begin{aligned}
\sum_{i=1}^{r} a_{i}^{\prime} A_{1}+\sum_{j=1}^{s} b_{j}^{\prime} B_{j} & \leq(1-\delta)\left(\sum_{i=1}^{r} a_{i} A_{i}+c \sum_{j=1}^{s} b_{j} B_{j}\right) \\
& =(1-\delta)(\Delta+c D) \leq \Delta+(1-\delta) c D .
\end{aligned}
$$

Thus, $c=\operatorname{fst}(X, \Delta ; D) \leq(1-\delta) c$, a contradiction.

As an immediate consequence, we obtain:

Proposition 3.3. Let $X$ be an F-finite noetherian normal scheme of characteristic $p>0$ and let $\Delta$ be an $\mathbb{R}$-divisor such that $(X, \Delta)$ is $F$ pure. Let $D$ be an effective $\mathbb{R}$-divisor on $X$ and let $c=\operatorname{fpt}(X, \Delta ; D)$. 
Then $(X, \Delta+c D)$ is F-pure.

\section{Non-globally-F-SPlit klt Del Pezzo surfaces}

The goal of this section is to prove Theorem 1.1. To this end, we first construct an unbounded sequence of klt del Pezzo surfaces, starting from a fixed $\log$ Calabi-Yau pair $\left(Z, C_{Z}\right)$, where $C_{Z}$ is a prime divisor and such that $Z$ admits exactly four singular points along $C_{Z}$. The construction was inspired by the "hunt" in [KM99. Note, in particular, that it holds over any algebraically closed field.

Proposition 4.1. Let $k$ be an algebraically closed field and let

$$
Q_{1}, \ldots, Q_{4} \in \mathbb{P}_{k}^{1}
$$

be four distinct closed points.

Then, there exists a two-dimensional projective plt pair $\left(Z, C_{Z}\right)$, where $C_{Z}$ is a prime divisor such that the following properties hold:

(1) $2\left(K_{Z}+C_{Z}\right) \sim 0$,

(2) there exists an isomorphism $\iota: C_{Z} \stackrel{\simeq}{\rightarrow} \mathbb{P}_{k}^{1}$ such that

$$
\iota_{*} \operatorname{Diff}_{C_{Z}}=\frac{1}{2} \sum_{i=1}^{4} Q_{i},
$$

and

(3) for every $\epsilon \in(0,1)$, there exist birational morphisms of projective klt surfaces

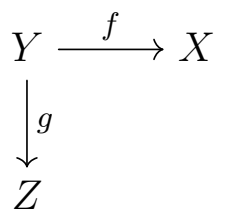

such that $-K_{X}$ is ample, the strict transform $C_{Y}$ of $C_{Z}$ on $Y$ coincides with the exceptional locus of $f$, and there exists $b \in(1-\epsilon, 1)$ such that $K_{Y}+b C_{Y}=f^{*} K_{X}$.

Proof. We divide the proof into three steps:

Step 1. We first construct $\left(Z, C_{Z}\right)$ and prove (1) and (2).

Let $C \simeq \mathbb{P}_{k}^{1}$ be the diagonal of $\mathbb{P}_{k}^{1} \times \mathbb{P}_{k}^{1}$ and let

$$
\begin{array}{ll}
F_{1}:=\pi_{1}^{*}\left(Q_{1}\right), & F_{2}:=\pi_{1}^{*}\left(Q_{2}\right), \\
F_{3}:=\pi_{2}^{*}\left(Q_{3}\right), & F_{4}:=\pi_{2}^{*}\left(Q_{4}\right),
\end{array}
$$

where $\pi_{i}: \mathbb{P}_{k}^{1} \times \mathbb{P}_{k}^{1} \rightarrow \mathbb{P}_{k}^{1}$ denotes the $i$-th projection, for $i=1,2$ (see Figure (1). 
In particular, the plt pair

$$
\left(\mathbb{P}_{k}^{1} \times \mathbb{P}_{k}^{1}, C+\frac{1}{2} \sum_{i=1}^{4} F_{i}\right)
$$

satisfies $2\left(K_{\mathbb{P}_{k}^{1} \times \mathbb{P}_{k}^{1}}+C+\frac{1}{2} \sum_{i=1}^{4} F_{i}\right) \sim 0$.

Let

$$
\varphi: S \longrightarrow \mathbb{P}_{k}^{1} \times \mathbb{P}_{k}^{1}
$$

be the blow-up along the four points in the set

$$
\left(F_{1} \cup F_{2}\right) \cap\left(F_{3} \cup F_{4}\right) .
$$

By abuse of notation, we denote the proper transforms of $C, F_{1}, \ldots, F_{4}$ on $S$ by the same symbols. We have

$$
2\left(K_{S}+C+\frac{1}{2} \sum_{i=1}^{4} F_{i}\right) \sim 0 .
$$

Note that $F_{1}, \ldots, F_{4}$ are pairwise disjoint $(-2)$-curves on $S$. Let

$$
\psi: S \rightarrow Z
$$

be the contraction of these curves and let $C_{Z}:=\psi_{*} C$. We obtain

$$
2\left(K_{Z}+C_{Z}\right)=\psi_{*}\left(2\left(K_{S}+C+\frac{1}{2} \sum_{i=1}^{4} F_{i}\right)\right) \sim 0 .
$$

Further, $\left(Z, C_{Z}\right)$ is plt and $\operatorname{Diff}_{C_{Z}}=\frac{1}{2} \sum_{i=1}^{4} Q_{i}$. Thus, (1) and (2) follow.

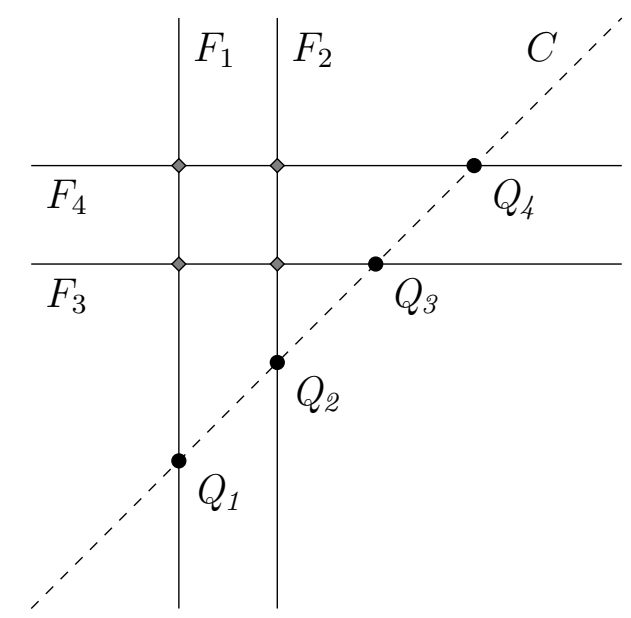

Figure 1. Curves on $\mathbb{P}_{k}^{1} \times \mathbb{P}_{k}^{1}$ 


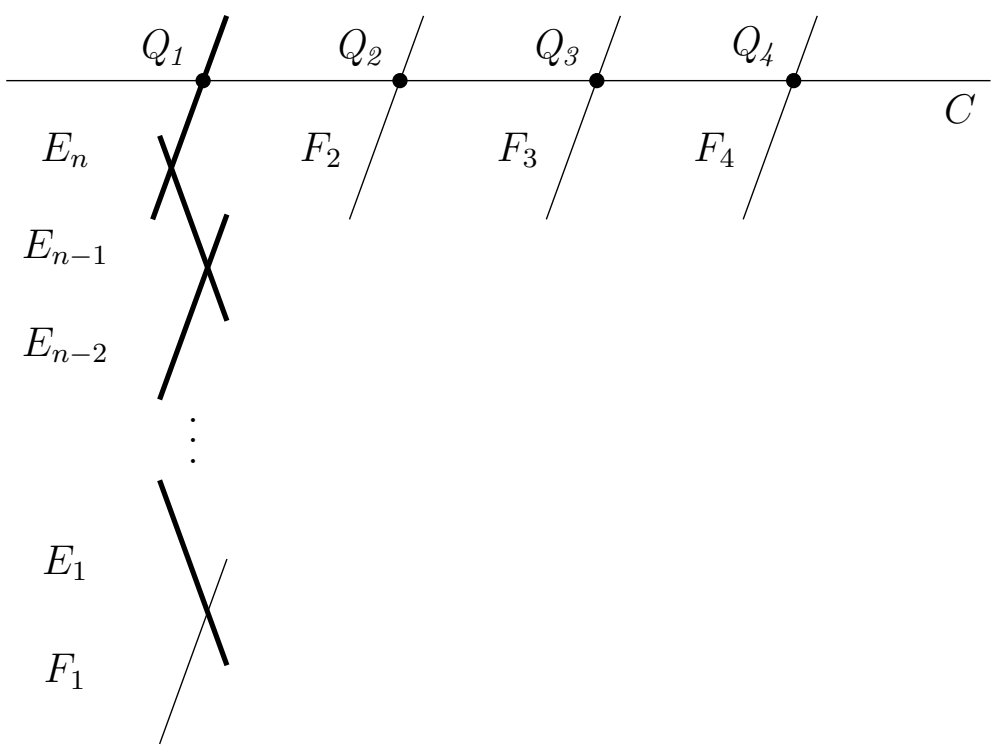

Figure 2. Curves on $\bar{S}$

Note that, by the Nakai-Moishezon criterion, $C_{Z}$ is ample. Moreover, $Z$ has exactly four singular points, and all of them lie on the curve $C_{Z}$.

Step 2. We now construct $X$ and $Y$.

Fix an integer $n \in \mathbb{Z}$ such that

$$
n \geq 4 \quad \text { and } \quad \frac{2}{n-\frac{7}{2}}<\epsilon .
$$

Let $\bar{S} \rightarrow S$ be the birational morphism obtained by blowing-up $n$-times the point $Q_{1} \in C$ : by the point $Q_{1}$ in the blow-up of $S$, we mean the unique intersection of the strict transform of $C$ with the exceptional curve. Again, by abuse of notation, we denote the strict transforms of $C, F_{1}, \ldots, F_{4}$ by the same symbols. Further, we denote the exceptional curves on $\bar{S}$ by $E_{1}, \ldots, E_{n}$, where $E_{i}$ is the exceptional curve of the $i$-th blow-up (see Figure 2).

It follows that

$$
2\left(K_{\bar{S}}+C+\frac{1}{2} \sum_{i=1}^{4} F_{i}+\frac{1}{2} \sum_{i=1}^{n} E_{i}\right) \sim 0 .
$$

Let

$$
h: \bar{S} \longrightarrow Y
$$

be the birational contraction of $F_{2}, F_{3}, F_{4}$ and of the chain of curves $E_{n-1}, \ldots, E_{1}, F_{1}$. We can construct such a birational morphism by 
running the MMP over $Z$ [Tan14, Theorem 6.5] with respect to the pair

$$
\left(\bar{S}, C+\frac{1}{2} E_{n}+\frac{3}{4}\left(\sum_{i=1}^{4} F_{i}+\sum_{i=1}^{n-1} E_{i}\right)\right) .
$$

Let $C_{Y}:=h_{*} C$ and $E_{Y}:=h_{*} E_{n}$. Then, there exists a birational morphism $g: Y \rightarrow Z$, whose exceptional divisor coincides with $E_{Y}$. In particular, $C_{Y}$ is the strict transform of $C_{Z}$ on $Y$. Thus, $C_{Y}$ is a smooth rational curve and it can be easily checked that

$$
C_{Y}^{2}=\frac{7}{2}-n
$$

Because of our choice of $n$, we have $C_{Y}^{2}<0$. We denote by $f: Y \rightarrow X$ the contraction of $C_{Y}$. Summarising, we have the following diagram:

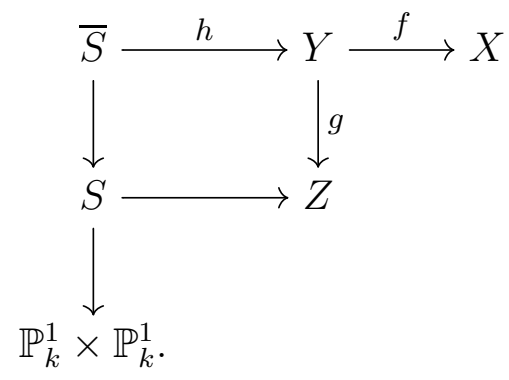

By taking a pullback to $\bar{S}$, it is easy to check that

$$
K_{Y}+C_{Y}+\frac{1}{2} E_{Y}=g^{*}\left(K_{Z}+C_{Z}\right) \sim_{\mathbb{Q}} 0 .
$$

Since $\left(Z, C_{Z}\right)$ is plt, we have that also $\left(Y, C_{Y}+\frac{1}{2} E_{Y}\right)$ is plt, and $Y$ is klt.

Step 3. We now prove (3).

Let $a \in \mathbb{Q}_{>0}$ be such that

$$
g^{*} C_{Z}=C_{Y}+a E_{Y} .
$$

Note that

$$
g^{*} C_{Z}-\delta E_{Y}=C_{Y}+(a-\delta) E_{Y}
$$

is ample for any $0<\delta \ll 1$, and so $f_{*} E_{Y}$ is ample. Since

$$
-\left(K_{Y}+C_{Y}\right) \sim_{\mathbb{Q}} \frac{1}{2} E_{Y},
$$

we have that

$$
-K_{X}=-f_{*}\left(K_{Y}+C_{Y}\right) \sim_{\mathbb{Q}} \frac{1}{2} f_{*} E_{Y}
$$


is ample. Let $b \in \mathbb{Q}$ be such that

$$
K_{Y}+b C_{Y}=f^{*} K_{X} .
$$

By intersecting with $C_{Y}$, we have

$$
b=\frac{K_{Y} \cdot C_{Y}}{-C_{Y}^{2}}=1-\frac{-\left(K_{Y}+C_{Y}\right) \cdot C_{Y}}{-C_{Y}^{2}} .
$$

Since $K_{Y}+C_{Y}+\frac{1}{2} E_{Y} \equiv 0$ and $C_{Y} \cdot E_{Y}>0$, we have

$$
0<-\left(K_{Y}+C_{Y}\right) \cdot C_{Y} \leq 2 .
$$

Thus, because of our choice of $n$, we obtain

$$
0<1-b=\frac{-\left(K_{Y}+C_{Y}\right) \cdot C_{Y}}{-C_{Y}^{2}} \leq \frac{2}{n-\frac{7}{2}}<\epsilon .
$$

In particular, $X$ is klt and (3) follows.

We can now prove our main theorem:

Proof of Theorem 1.1. Fix an algebraically closed field $k$ of characteristic $p>0$. If $p=2$, then the smooth cubic surface of Fermat type satisfies the required properties [Har98a, Example 5.5]. Thus, we may assume $p \geq 3$.

Let $Q_{1}, \ldots, Q_{4} \in \mathbb{P}_{k}^{1}$ be as in Lemma 2.4 and let $X, Y, Z$ and $b$ be as in Proposition 4.1 for some $0<\epsilon \ll 1$. In particular, $X$ is klt. By (1) and (2) of Proposition 4.1 and Lemma 2.5, it follows that the pair $\left(Z, C_{Z}\right)$ is not globally $F$-split. By Theorem 3.2 , there exists a rational number $\beta$, independent of $\epsilon$, such that $0<\beta<1$ and the pair $\left(Z, \beta C_{Z}\right)$ is not globally $F$-split as well.

Thus, we may assume $\epsilon<1-\beta$, and in particular $b>\beta$. Since $\left(Z, \beta C_{Z}\right)$ is not globally $F$-split, Lemma 2.2 implies that $\left(Y, \beta C_{Y}\right)$ is not globally $F$-split. Thus, $\left(Y, b C_{Y}\right)$ is not globally $F$-split and, by Lemma 2.3, also $X$ is not globally $F$-split.

\section{NON-F-PURE CANONICAL THREEFOLDS}

The goal of this section is to prove Theorem [1.2, We begin with the following Lemma.

Lemma 5.1. Let $k$ be an algebraically closed field of characteristic $p>5$. Let $f(x, y, z) \in k[x, y, z]$ be a homogeneous cubic polynomial such that $\operatorname{Proj} k[x, y, z] /(f(x, y, z))$ is a smooth elliptic curve.

Then, for every $n \in \mathbb{Z}_{\geq 0}$,

$$
X_{n}=\operatorname{Spec} k[x, y, z, w] /\left(f(x, y, z)+w^{n}\right)
$$

has at most canonical singularities. 
Proof. We divide the proof into three steps:

Step 1. We first show that $X_{n}$ is smooth outside the origin and, in particular, $X_{n}$ is smooth if $n \leq 1$.

Let $(a, b, c, d) \in X_{n} \subset \mathbb{A}_{k}^{4}$ be a singular point. Since $f$ is homogeneous of degree 3 , we have

$$
x \frac{\partial f}{\partial x}+y \frac{\partial f}{\partial y}+z \frac{\partial f}{\partial z}=3 f .
$$

Since $p>3$, a singular point $(a, b, c, d)$ satisfies the following equation:

$$
f(a, b, c)=\frac{\partial f}{\partial x}(a, b, c)=\frac{\partial f}{\partial y}(a, b, c)=\frac{\partial f}{\partial z}(a, b, c)=0 .
$$

This implies $a=b=c=0$, hence $d=0$, as claimed.

Step 2. We now show that $X_{2}$ is terminal.

Since $p>3$, by writing the cubic $f$ in Weierstrass form, we may assume that there exist $a, b \in k$ such that

$$
f(x, y, z)+w^{2}=y^{2} z+x^{3}+a x z^{2}+b z^{3}+w^{2} .
$$

After taking a linear transformation $x \mapsto x+c z$ for some $c \in k$, we may write

$$
f(x, y, z)+w^{2}=y^{2} z+x^{3}+\alpha x^{2} z+\beta x z^{2}+\gamma z^{3}+w^{2}
$$

for some $\alpha, \beta, \gamma \in k$ with $\gamma \neq 0$. Consider the hypersurface $S$ in $X_{2}$ obtained by cutting with the hyperplane $\{x=0\}$, i.e.

$$
S=\operatorname{Spec} k[y, z, w] /\left(y^{2} z+\gamma z^{3}+w^{2}\right) .
$$

Then $S$ admits a unique singularity which is du Val of type $D_{4}$, and in particular $S$ is canonical. Since $p>5, S$ is strongly $F$-regular Har98b, Theorem 1.1] and inversion of adjunction [Das15, Theorem A] implies that the pair $\left(X_{2}, S\right)$ is plt. Since $S$ is a Cartier divisor, it follows that $X_{2}$ is terminal, as claimed.

Step 3. We now show that $X_{n}$ is canonical for all $n \in \mathbb{Z}_{\geq 0}$.

We may assume $n \geq 3$. Consider the blow-up $f: Y \rightarrow X_{n}$ at the origin. Then $Y$ is covered by four affine open subsets:

$$
\begin{aligned}
Y & =\operatorname{Spec} k[x, y, z, w] /\left(f(1, y, z)+x^{n-3} w^{n}\right) \\
& \cup \operatorname{Spec} k[x, y, z, w] /\left(f(x, 1, z)+y^{n-3} w^{n}\right) \\
& \cup \operatorname{Spec} k[x, y, z, w] /\left(f(x, y, 1)+z^{n-3} w^{n}\right) \\
& \cup \operatorname{Spec} k[x, y, z, w] /\left(f(x, y, z)+w^{n-3}\right) . \\
&
\end{aligned}
$$


We first show that the first three affine open subsets are smooth. By symmetry, it is enough to consider the first one. By Step 1, we just need to consider points in the exceptional locus $\{x=0\}$. Since $n \geq 3$, a singular point $(x, y, z, w)$ satisfies the equations:

$$
f(1, y, z)=\frac{\partial f(1, y, z)}{\partial y}=\frac{\partial f(1, y, z)}{\partial z}=0 .
$$

Since Spec $k[y, z] /(f(1, y, z))$ is a smooth affine curve, there are no such points. Thus, the singular locus of $Y$ is contained in the last affine open subset

$$
\text { Spec } k[x, y, z, w] /\left(f(x, y, z)+w^{n-3}\right) \simeq X_{n-3}
$$

Moreover, the multiplicity of $X_{n}$ at the origin is three. Thus, $f$ is crepant, i.e.

$$
K_{Y}=f^{*} K_{X_{n}}
$$

Thus, it is enough to show that $X_{n-3}$ is canonical. Repeating the same procedure finitely many times, Step 1 and Step 2 imply the claim. This completes the proof of the Lemma.

Lemma 5.2. Let $k$ be an algebraically closed field of characteristic $p>0$. Let $f(x, y, z) \in k[x, y, z]$ be a homogeneous cubic polynomial such that Proj $k[x, y, z] /(f(x, y, z))$ is a smooth elliptic curve.

Let $n \in \mathbb{Z}$ be such that $n \geq p$, and let

$$
X_{n}=\operatorname{Spec} k[x, y, z, w] /\left(f(x, y, z)+w^{n}\right) .
$$

Then, $X_{n}$ is F-pure if and only if Proj $k[x, y, z] /(f(x, y, z))$ is an ordinary elliptic curve.

Proof. We consider the following conditions.

(1) Proj $k[x, y, z] /(f(x, y, z))$ is an ordinary elliptic curve.

(2) Spec $k[x, y, z] /(f(x, y, z))$ is $F$-pure.

(3) $f(x, y, z)^{p-1} \notin\left(x^{p}, y^{p}, z^{p}\right) k[x, y, z]$.

(a) $X_{n}$ is $F$-pure.

(b) $\left(f(x, y, z)+w^{n}\right)^{p-1} \notin\left(x^{p}, y^{p}, z^{p}, w^{p}\right) k[x, y, z, w]$.

It is well known that (1), (2) and (3) (respectively, (a) and (b)) are equivalent. Since, by assumption: $n \geq p$, it is easy to check that (3) and (b) are equivalent. Thus, the claim follows.

We can now prove Theorem 1.2 . Proof of Theorem 1.2. If $p \leq 5$, then

$$
X:=\operatorname{Spec} k[x, y, z, w] /\left(x^{2}+y^{3}+z^{5}\right)
$$

is canonical but not $F$-pure [Har98b, (4.4)]. 
Thus, we may assume $p>5$. Let $f(x, y, z) \in k[x, y, z]$ be a homogeneous cubic polynomial such that $\operatorname{Proj} k[x, y, z] /(f(x, y, z))$ is a supersingular elliptic curve. Let

$$
X:=k[x, y, z, w] /\left(f(x, y, z)+w^{p}\right) .
$$

By Lemma 5.1, $X$ is canonical. By Lemma 5.2, $X$ is not $F$-pure.

\section{REFERENCES}

[BMS08] M. Blickle, M. Mustaţă, and K.E. Smith, Discreteness and rationality of F-thresholds, Michigan Math. J. 57 (2008), 43-61, Special volume in honor of Melvin Hochster.

[BSTZ10] M. Blickle, K. Schwede, S. Takagi, and W. Zhang, Discreteness and rationality of F-jumping numbers on singular varieties, Math. Ann. 347 (2010), no. 4, 917-949.

[CGS14] P. Cascini, Y. Gongyo, and K. Schwede, Uniform bounds for strangly F-regular surfaces, Trans. Amer. Math. Soc. (to appear) (2014).

[CTW15] P. Cascini, H. Tanaka, and J. Witaszek, On log del Pezzo surfaces in large characteristic, Preprint (2015).

[Das15] O. Das, On strongly F-regular inversion of adjunction, J. Algebra 434 (2015), 207-226.

[Har98a] N. Hara, A characterization of rational singularities in terms of injectivity of Frobenius maps, Amer. J. Math. 120 (1998), no. 5, 981-996.

[Har98b] N. Hara, Classification of two-dimensional F-regular and F-pure singularities, Adv. Math. 133 (1998), no. 1, 33-53.

[Har06] F- Fure thresholds and F-jumping exponents in dimension two, Math. Res. Lett. 13 (2006), no. 5-6, 747-760, With an appendix by Paul Monsky.

[Her12] D.J. Hernández, F-purity of hypersurfaces, Math. Res. Lett. 19 (2012), no. 2, 389-401.

[HH90] M. Hochster and C. Huneke, Tight closure, invariant theory, and the Briançon-Skoda theorem, J. Amer. Math. Soc. 3 (1990), no. 1, 31-116.

[HNBWZ15] D.J. Hernández, L. Núñez-Betancourt, E.E. Witt, and W. Zhang, Fpure thresholds of homogeneous polynomials, Michigan Math. J. (to appear) (2015).

[HX15] C. Hacon and C. Xu, On the three dimensional minimal model program in positive characteristic, J. Amer. Math. Soc. 28 (2015), no. 3, 711-744.

$\left[\mathrm{K}^{+} 92\right] \quad$ J. Kollár et al., Flips and abundance for algebraic threefolds, Société Mathématique de France, Paris, 1992.

[KM98] J. Kollár and S. Mori, Birational Geometry of Algebraic Varieties, Cambridge Tracts in Mathematics, vol. 134, Cambridge University Press, 1998.

[KM99] S. Keel and J. McKernan, Rational curves on quasi-projective surfaces, Mem. Amer. Math. Soc. 140 (1999), no. 669, viii+153.

[PST14] Z. Patakfalvi, K. Schwede, and K. Tucker, Notes for the workshop on positive characteristic algebraic geometry, arXiv:1412.2203 (2014). 
[Sch08] K. Schwede, Generalized test ideals, sharp F-purity, and sharp test elements, Math. Res. Lett. 15 (2008), no. 6, 1251-1261.

[Smi00] K. E. Smith, Globally F-regular varieties: applications to vanishing theorems for quotients of Fano varieties, Michigan Math. J. 48 (2000), 553-572, Dedicated to William Fulton on the occasion of his 60th birthday.

[SS10] K. Schwede and K. E. Smith, Globally F-regular and log Fano varieties, Adv. Math. 224 (2010), no. 3, 863-894.

[Tan14] H. Tanaka, Minimal models and abundance for positive characteristic log surfaces, Nagoya Math. J. 216 (2014), 1-70.

[TW04] S. Takagi and K. Watanabe, On F-pure thresholds, J. Algebra 282 (2004), no. 1, 278-297.

[Wat91] K. Watanabe, F-regular and F-pure normal graded rings, J. Pure Appl. Algebra 71 (1991), no. 2-3, 341-350.

Department of Mathematics, Imperial College, London, 180 Queen's Gate, London SW7 2AZ, UK

E-mail address: p.cascini@imperial.ac.uk

E-mail address: h.tanaka@imperial.ac.uk

E-mail address: j.witaszek14@imperial.ac.uk 